as nine others of a sort excluded from the present consideration, namely perspective transformations. It is of interest to observe that the three other inflexional triangles of each invariant cubic of the third class are themselves members of the system, and are therefore transformed into themselves, not interchanged, by the eight non-perspective collineations of the cubic.

Of plane curves of higher order than the third, it is easily shown that only highly specialized classes are collinear with themselves, and that of these classes the groups are correspondingly small. It appears possible, however, to extend this method of inquiry to such interesting topics as these :

(1) What simultaneous invariant conditions must two non-singular collineations satisfy in order to belong to the group leaving a common conic unaltered?

(2) What invariant conditions are met by collineations which leave unaltered a quadric surface? a twisted cubic? a twisted quartic curve?

NoRTH WESTERN UNIVERSITY, August, 1897.

\title{
A GENERATING FUNCTION FOR THE NUMBER OF PERMUTATIONS WITH AN ASSIGNED NUMBER OF SEQUENCES.
}

BY PROFESSOR F. MORLEY.

(Read before the American Mathematical Society at the Meeting of May 29, 1897.)

\$1. André's Recurrence-formula. In Liouville's Journal, 1895, and in earlier memoirs there referred to, M. André proves the formula

$$
P_{n, s}=s P_{n 1, s}+2 P_{n-1, s-1}+(n-s) P_{n-1, s-2}
$$

where $P_{n, s}$ is the number of permutations of $n$ things (say of the numbers $1,2, \cdots n$ ) with $s$ sequences; and shows that (taking the number of sequences as great as possible) the numbers $\frac{1}{2} P_{n+1, n}$ are the coefficients of $x^{n} / n !$ in $1 /(1-\sin x)$, when expressed as a Maclaurin series.

My object is to obtain a function of $x$ and $y$ which when developed in positive integer powers of $x$ and $y$ will have $P_{n, \text { as }}$ the general coefficient. 
It is convenient to replace the consideration of sequences by that of runs, where I define a run as three adjacent numbers in order of magnitude (ascending or descending). If there are $m$ numbers in order of magnitude they give $m-2$ runs, but one sequence. Whenever, by rearranging the numbers, we gain a run, we lose a sequence, so that if in a permutation of $n+1$ things there are $r$ runs and $s$ sequences then

$$
r+s=\text { constant }
$$

or since in the natural order there are $n-1$ runs and one sequence

$$
r+s=n \text {. }
$$

Let $c_{r, n}$ be half the number of permutations of the $n+1$ things, when there are $r$ runs; that $c_{r, n}$ is an integer is clear because any permutation when read backwards yields another with the same number of runs. We have then, rewriting André's formula

$$
c_{r, n}=(n-r) c_{r-1, n-1}+2 c_{r, n-1}+(r+1) c_{r+1, n-1} .
$$

The initial values are known from the definition, to be

\begin{tabular}{|c|c|c|c|c|c|}
\hline$r=$ & 0 & 1 & 2 & 3 & 4 \\
\hline$n=1$ & 1 & & & & \\
\hline 2 & 2 & 1 & & & \\
\hline 3 & 5 & 6 & 1 & & \\
\hline 4 & 16 & 29 & 14 & 1 & \\
\hline 5 & 61 & 150 & 118 & 30 & 1 \\
\hline
\end{tabular}
$c_{0,1}=1, c_{0,2}=2, c_{1,2}=1$; and so on; whence using for the higher values the recurrence formula we have the table:

It must be noticed that in accordance with this table we have $c_{-1, n}=0$ for any positive $n$.

\$2. The differential equation. There is, of course, freedom in the choice of a double Maclaurin series which shall have as its cofficients the numbers $c_{r, n}$. Thus we can take as the typical term

$$
c_{r, n} x^{r} y^{n}, c_{r, n} x^{r} y^{n} / r !, c_{r, n} x^{r} y^{n} / n !, \text { or } c_{r, n} x^{r} y^{n} / r ! n !
$$


Of these the third turns out to be the most convenient. Let then

$$
z=\sum_{r=0}^{\infty} \sum_{n=1}^{\infty} c_{r, n} x^{r} y^{n} / n !
$$

Then

$$
D_{y} z=\sum_{r=0} \sum_{n=1} c_{r, n} x^{r} y^{n-1} /(n-1) !
$$

or from (2)

$$
\begin{gathered}
D_{y} z=\sum \sum\left[2 c_{r, n-1}+(r+1) c_{r+1, n-1}\right. \\
\left.+(n-r) c_{r-1, n-1}\right] x^{r} y^{n-1} /(n-1) ! \\
=2 \sum_{r=0} \sum_{n=0} c_{r, n} x^{r} y^{n} / n !+\sum_{r=1} \sum_{n=0} c_{r, n} r x^{r-1} y^{n} / n ! \\
-\sum_{r=-1} \sum_{n=0} c_{r, n} r x^{r+1} y^{n} / n !+\sum_{r=-1} \sum_{n=0} c_{r, n} x^{r+1} y^{n} /(n-1) ! \\
=2(z+P x)+D_{x}(z+P x)-x^{2} D_{x}(z+P x)+x y D_{y}(z+P x),
\end{gathered}
$$

where $P x$ is the series $\sum_{r=0}^{\infty} c_{r, 0} x^{r}$. We have then, if $z_{1}=z+P x$, the equation

$$
(1-x y) D_{y} z_{1}-\left(1-x^{2}\right) D_{x} z_{1}=2 z_{1} .
$$

§3. Solution of the differential equation. We have to solve

$$
\frac{d y}{1-x y}=-\frac{d x}{1-x^{2}}=\frac{d z_{1}}{2 z_{1}}
$$

From the second and third expressions

$$
z_{1} \frac{1+x}{1-x}=\text { a constant } a .
$$

To solve $\frac{d y}{1-x y}=-\frac{d x}{1-x^{2}}$ write $x=\sin t$.

Then $\quad d y \cos t=-(1-y \sin t) d t$

or

$$
y \cos t+t=b \text {. }
$$

Hence the solution of (4) is

$$
z_{1} \frac{1+x}{1-x}=f(y \cos t+t) \text {. }
$$


Now André has proved that when $x=0$,

$$
z=\frac{1}{1-\sin y}-1
$$

Hence the form of the function $f$ is known; and we have

$$
(z+P x) \frac{1+x}{1-x}=\frac{1}{1-\sin (y \cos t+t)} .
$$

We determine $P x$ by the condition that $z=0$ when $y=0$. This gives

$$
P x \frac{1+x}{1-x}=\frac{1}{1-\sin t}=\frac{1}{1 \quad x} .
$$

Hence finally the generating function is

$$
z=\frac{1-x}{1+x} \frac{1}{1-\sin (y \cos t+t)}-\frac{1}{1+x}
$$

where $t$ is that arc whose sine is $x$ which vanishes with $x$.

As a test of correctness let $x=1$. The series (3) is then

$$
\begin{aligned}
z & =\frac{1}{2}\left(2 ! y+3 ! y^{2} / 2 !+4 ! y^{3} / 3 !+\cdots\right) \\
& =\frac{1}{2}\left(\frac{1}{(1-y)^{2}}-1\right) .
\end{aligned}
$$

And the limit for the function (5) is

$$
\begin{aligned}
& \frac{1}{2}\left(\lim _{t=\pi / 2} \frac{1-\sin t}{1-\sin (y \cos t+t)}-1\right) \\
= & \frac{1}{2}\left(\lim _{\epsilon=0} \frac{1-\cos \varepsilon}{1-\cos (\varepsilon-y \sin \varepsilon)}-1\right) \\
= & \frac{1}{2}\left(\frac{1}{(1-y)^{2}}-1\right) .
\end{aligned}
$$

\$4. The polynomials in $x$. As an application of (5), we can expand directly in powers of $y$ and thus examine the polynomials $Q_{n} x$ which is the coefficient of $y^{n} / n !$, this polynomial being of degree $n$. 
We have

$$
\begin{aligned}
Q_{n} x & =\frac{1-x}{1+x} \lim _{y=0} D_{y}^{n} \frac{1}{1-\sin (y \cos t+t)} \\
& =\frac{1-x}{1+x} \cos ^{n} t D_{t}^{n} \frac{1}{1-\sin t}
\end{aligned}
$$

From this form the polynomials are readily investigated. It may be remarked that, since

$$
\frac{1}{1-\sin t}=\frac{1}{1-\exp i(t-\pi / 2)}-\left(\frac{1}{1-\exp i(t-\pi / 2)}\right)^{2}
$$

the explicit determination of

$$
D_{t}^{n} \frac{1}{1-\sin t}
$$

reduces to that of

$$
D_{u}^{n} \frac{1}{1-e^{u}}
$$

Let this expression be

$$
\frac{A_{1}}{1-e^{u}}+\frac{A_{2}}{\left(1-e^{u}\right)^{2}}+\cdots+\frac{A_{n+1}}{\left(1-e^{u}\right)^{n+1}}
$$

then considering only negative powers of $u$ we have

$$
P u+(-)^{n+1} n ! / u^{n+1} \equiv \frac{A_{1}}{1-e^{u}}+\cdots+\frac{A_{n+1}}{\left(1-e^{u}\right)^{n+1}},
$$

whence the coefficients $A$ are to be found; and writin $1-e^{u}=v$ we have

$$
\frac{A_{n+1}}{v^{n+1}}+\frac{A_{n}}{v^{n}}+\cdots+\frac{A_{1}}{} \equiv \frac{(-)^{n+1} n !}{(\log (1-v))^{n+1}}+P v
$$

whence, so far as the $n^{\text {th }}$ power of $v$,

$$
A_{n+1}+A_{n} v+\cdots+A_{1} v^{n} \equiv \frac{n !}{\left(1+v / 2+v^{2} / 3+\cdots\right)^{n+1}}
$$

that is the coefficients $A$ are determined as the coefficients of the first $n$ powers in

$$
n !\left(1+v / 2+v^{2} / 3+\cdots\right)^{-n 1}
$$


Formula (6) shows at once that when $n$ is even (say $n=2 m), Q_{n}$ is exactly divisible by $(1+x)^{m-1}$. For the numerator has the factor $\left(1-x^{2}\right)^{m}$, and the denominator is $(1+x)(1-x)^{2 m+1}$. Similarly when $n=2 m-1, Q_{n}$ is divisible by $(1+x)^{m-1}$; for $D_{t}^{n} \frac{1}{1-\sin t}$ has now the factor cos $t$ in the numerator, whence $Q_{n}$ has still in its numerator the factor $\left(1-x^{2}\right)^{m}$. For example, from the table

$$
Q_{5} x=61+150 x+118 x^{2}+30 x^{3}+x^{4}
$$

and this is exactly divisible by $(1+x)^{2}$.

HAVERFORD COLLEGE,

June, 1897.

\section{LINE GEOMETRY.}

La Géométrie réglée et ses applications. Par G. Konnigs. Paris, Gauthier-Villars et Fils. 1895. 4to. 146 pp.

The first general impulse to the study of the geometry of the straight line was given by Plücker's posthumous work in 1868-9, which was soon followed by a large number of contributions from German, French and Italian mathematicians ; the English people were soon interested in the application of the new geometry to mechanics and physics.

In the work of Plücker, point and plane coördinates are used nearly throughout the book, and in many cases the notation is so complicated that readers frequently lose interest before the most important parts are reached.

In most of the subsequent contributions the idea of point or plane coördinates is not considered; most of them presuppose a knowledge of the relation between these and the quantities which directly define a straight line, while others define the new coördinates as parameters, without discussing their meaning.

In view of the great interest which the subject has awakened and its fruitful application to the study of curves and surfaces, its analogy to the geometry of the sphere and its assistance to mechanics, one wonders why no elementary and systematic treatise on the subject has appeared. The work of Sturm is the only comprehensive work on line geometry that has as yet appeared, and it is by no means an elementary one. It is purely synthetic in its treatment 